\title{
Density and Uniqueness in Percolation
}

\author{
R. M. Burton ${ }^{1}$ and M. Keane ${ }^{2}$ \\ ${ }^{1}$ Department of Mathematics, Oregon State University, Corvallis, OR 97331, USA \\ 2 Department of Mathematics and Informatics, Delft University of Technology, \\ NL-2628 BL Delft, The Netherlands
}

\begin{abstract}
Two results on site percolation on the $d$-dimensional lattice, $d \geqq 1$ arbitrary, are presented. In the first theorem, we show that for stationary underlying probability measures, each infinite cluster has a well-defined density with probability one. The second theorem states that if in addition, the probability measure satisfies the finite energy condition of Newman and Schulman, then there can be at most one infinite cluster with probability one. The simple arguments extend to a broad class of finite-dimensional models, including bond percolation and regular lattices.
\end{abstract}

Our notation is as follows. Let $\mathbf{Z}^{\mathbf{d}}$ be the $d$-dimensional lattice, with $d \geqq 1$. Finite $d$-dimensional boxes in $\mathbf{Z}^{\mathbf{d}}$ whose sides are parallel to the coordinate directions are called rectangles. The number of points in a finite set $F$ is denoted by \# $(F)$. We set

$$
X=\{0,1\}^{\mathbf{Z}^{\mathbf{d}}},
$$

and we let $\mu$ denote a probability measure on $X$ which is stationary, i.e. invariant under translation by each element of $\mathbf{Z}^{\mathbf{d}}$. For each $x \in X$, the connected components of the nearest neighbor graph whose set of vertices is

$$
\left\{z \in \mathbf{Z}^{\mathbf{d}}: x(z)=\mathbf{1}\right\}
$$

are called clusters.

Definition. A subset $S$ of $\mathbf{Z}^{\mathbf{d}}$ has density $\alpha$ if for each sequence of rectangles $R_{1} \cong R_{2} \subseteq \ldots$ with $\bigcup_{n \geqq 1} R_{n}=\mathbf{Z}^{\mathbf{d}}$, the limit

$$
\lim _{n \rightarrow \infty} \frac{\#\left(S \cap R_{n}\right)}{\#\left(R_{n}\right)}
$$

exists and is equal to $\alpha$. If $S$ does not have density [ $\alpha$ for any $\alpha$ ], then $S$ is rough.

Theorem 1. For $\mu$-almost every $x \in X$, each cluster of $x$ has density. 
Proof. By ergodic decomposition, we may assume without loss of generality that $\mu$ is ergodic. Then the number $N$ of infinite clusters of $x$ is $\mu$-almost surely constant, with $0 \leqq N \leqq \infty$. (We need only consider infinite clusters, since finite clusters clearly have density zero.)

Fix $x \in X$ with $N$ infinite clusters. If $R$ is any rectangle, each infinite cluster $C$ of $x$ has $\#(C \cap R)$ points in $R$; let

$$
m_{1}(R, x) \geqq m_{2}(R, x) \geqq \ldots
$$

designate the numbers \# $(C \cap R)$ for the different infinite clusters $C$ of $x$, arranged in some non-increasing order with their proper multiplicities. The rank of $C$ in $R$ is the index of this arrangement which corresponds to $C$; it may depend on $R$.

For each $K<N+1$ we define a stochastic process $F$ by

$$
F_{R}(x)=m_{1}(R, x)+\ldots+m_{K}(R, x),
$$

indexed by rectangles, with underlying probability space $(X, \mu)$. Since $F$ is subadditive [that is, if any rectangle is partitioned into a disjoint union of smaller rectangles, then the value of $F$ on the large rectangle is smaller than or equal to the sum of the values of $F$ on the smaller rectangles, for each $x]$, the multiparameter subadditive ergodic theorem of Akcoglu and Krengel ([2], see also [8]) yields the existence of a constant $\gamma=\gamma(K) \geqq 0$ such that

$$
\lim _{n \rightarrow \infty} \frac{R_{R_{n}}}{\#\left(R_{n}\right)}=\gamma
$$

$\mu$-almost surely, for any rectangle sequence $R_{1} \cong R_{2} \cong \ldots$ with union equal to $\mathbf{Z}^{\mathrm{d}}$. Therefore the "density" of the rank $K$ cluster in $R$ exists and is equal to

$$
\alpha(K)=\gamma(K)-\gamma(K-1)
$$

[with $\gamma(0)=0$ ]; we write "density" because the rank of a fixed cluster $C$ of $x$ may vary with $R$.

Now suppose in addition that

$$
\lim _{n \rightarrow \infty} \frac{\#\left(R_{n+1} \backslash R_{n}\right)}{\#\left(R_{n}\right)}=0 ;
$$

this property can be obtained from any rectangle sequence by adding additional rectangles. Let $C$ be a fixed cluster of $x$ and let $K_{n}$ be the rank of $C$ in the rectangle $R_{n}$. We now can show that the sequence

$$
\frac{m_{K_{n}}\left(R_{n}, x\right)}{\#\left(R_{n}\right)}
$$

of densities of $C$ in the rectangles $R_{n}$ converges as $n$ tends to infinity, for $\mu$-almost all $x \in X$. On the one hand, the subadditivity statement above shows that the set of limit points of this sequence is contained in the set

$$
\{0\} \cup\{\alpha(K): K<N+1\}
$$


$\mu$-almost surely; on the other hand, this set of limit points must be a closed interval, because the difference between the terms $n$ and $n+1$ of the sequence is bounded by

$$
\frac{2 \#\left(R_{n+1} \backslash R_{n}\right)}{\#\left(R_{n}\right)},
$$

which tends to zero. Therefore the sequence has exactly one limit point, as the set

$$
\{0\} \cup\{\alpha(K): K<N+1\}
$$

contains no intervals of positive length. That is, $C$ has density, and the proof is finished.

We remark that by using the techniques of Grimmett [7], an easy proof for the subadditive theorem in the above situation is available, the point being that $F$ is also "asymptotically superadditive."

For our second result we first define the notion of finite energy. If $F$ is a finite subset of $\mathbf{Z}^{\mathbf{d}}$ and

$$
\phi: F \rightarrow\{0,1\}
$$

is a "coloring" of $F$, then we can associate to each $x \in X$ a point $\tilde{x} \in X$ by changing the values of $x$ on $F$ to $\phi$ :

$$
\tilde{x}(z)=\left\{\begin{array}{lll}
\phi(z) & \text { if } & z \in F \\
x(z) & \text { if } & z \notin F .
\end{array}\right.
$$

If $E \subseteq X$ is an event, we set $\phi(E)=\{\tilde{x}: x \in E\}$.

Definition 2. The probability measure $\mu$ has finite energy if for any $E \subseteq X$ with $\mu(E)>0$ and any $F$ and $\phi$,

$$
\mu(\phi(E))>0 .
$$

Theorem 2. If $\mu$ [is stationary and] has finite energy, then $\mu$-almost every $x \in X$ has at most one infinite cluster.

Proof. By ergodic decomposition, we may assume without loss of generality that $\mu$ is ergodic so that $N$, the total number of clusters, is constant $\mu$-almost surely. Then $N$ is either zero, one or infinity. (See [9]; the proof is a straightforward application of finite energy and ergodicity.) If $N$ is zero or one we are finished so assume $N$ is infinity. A point $z \in \mathbf{Z}^{\mathbf{d}}$ is an encounter point for $x \in X$ if the following two conditions hold:

(1) $z$ belongs to an infinite cluster $C$ of $x$, and

(2) the set $C \backslash\{z\}$ has no finite components and exactly three infinite components. Again using finite energy to change $x$ in a large box, it is seen that the origin is an encounter point with positive probability, say $2 \varepsilon$. It follows from the ergodic theorem that for $\mu$-almost all $x \in X$ all large (depending on $x$ ) rectangles $R$ contain at least $\varepsilon \#(R)$ encounter points.

We next show that for any $x \in X$ and any rectangle $R$, the number of encounter points of $x$ in $R$ is less than the number of points on the boundary of $R$. This will finish the proof, since we cannot have

$$
\varepsilon \#(R)<\# \text { (boundary of } R \text { ) }
$$

for large $R$, because the dimension $d$ is finite. 
Let $C$ be a fixed cluster of $x$. Define

$$
Y=C \cap \text { (boundary of } R \text { ). }
$$

If $z \in R$ is an encounter point for $x$, then removal of $z$ from $C$ defines by (2) a partition

$$
P=\left\{P_{1}, P_{2}, P_{3}\right\}
$$

of the set $Y$, such that $P_{i} \neq \emptyset$ for $1 \leqq i \leqq 3$. Moreover, if $z^{\prime} \in R$ is another encounter point with partition $Q=\left\{Q_{1}, Q_{2}, Q_{3}\right\}$, then the indices for $P$ and $Q$ can be chosen in such a way that $P_{1} \supseteqq Q_{2} \cup Q_{3}$ : simply choose $P_{1}$ to correspond to the component of $C \backslash\{z\}$ containing $z^{\prime}$ and $Q_{1}$ to correspond to the component of $C \backslash\left\{z^{\prime}\right\}$ containing $z$. Thus the following lemma implies that the number of encounter points belonging to $C$ is at most \#(Y)-2, and summing over all infinite clusters completes the proof.

For our final lemma we shall use the following notation. Let $Y$ be a finite set with at least three elements. A partition of $Y$ is a collection $P=\left\{P_{1}, P_{2}, P_{3}\right\}$ of three non-empty disjoint subsets of $Y$ whose union is $Y$. Partitions $P$ and $Q$ are compatible if there is an ordering of each such that $P_{1} \supseteqq Q_{2} \cup Q_{3}$. A collection $\mathbf{P}$ of partitions is compatible if each pair $P, Q \in \mathbf{P}$ is compatible.

Lemma. If $\mathbf{P}$ is a compatible collection of partitions of $Y$, then

$$
\#(\mathbf{P}) \leqq \#(\mathbf{Y})-\mathbf{2} \text {. }
$$

Proof. If \# $(Y)=3$, then $Y$ has only one partition and the lemma is valid. We proceed by induction on $n=\#(Y)$, supposing the lemma valid whenever $\#(Y)<n$. Choose any $P \in \mathbf{P}$. Then $P=\left\{P_{1}, P_{2}, P_{3}\right\}$ with $\#\left(P_{i}\right)=n_{i}>0$ and $n_{1}+n_{2}+n_{3}=n$. Divide $\mathbf{P} \backslash\{\mathbf{P}\}$ into three classes $\mathbf{P}_{\mathbf{i}}(1 \leqq i \leqq \mathbf{3})$, with $Q \in \mathbf{P}_{\mathbf{i}}$ if

$$
P_{i} \supseteqq Q_{2} \cup Q_{3}
$$

after reordering of the $Q$-indices. (The $P$-indices are now fixed.) Clearly each $Q \in \mathbf{P} \backslash\{\mathbf{P}\}$ belongs to exactly one $\mathbf{P}_{\mathbf{i}}$. Now fix an $i$, say $i=1$, and define

$$
Y_{1}=P_{1} \cup\{\Delta\},
$$

where $\Delta \notin Y$. For each $Q \in \mathbf{P}_{\mathbf{1}}$ define a partition $Q^{\prime}$ of $Y_{1}$ by setting $Q^{\prime}=\left\{Q_{1}^{\prime}, Q_{2}, Q_{3}\right\}$ with $Q_{1}^{\prime}=\left(Q_{1} \cap P_{1}\right) \cup\{\Delta\}$. Then

$$
\mathbf{P}_{\mathbf{1}}^{\prime}=\left\{\mathbf{Q}^{\prime}: \mathbf{Q} \in \mathbf{P}_{\mathbf{1}}\right\}
$$

is a compatible collection of partitions of $Y_{1}$, and

$$
\#\left(Y_{1}\right)=n_{1}+1<n_{1}+n_{2}+n_{3}=n \text {. }
$$

Hence by induction

$$
\#(\mathbf{P})=\sum \#\left(\mathbf{P}_{\mathbf{i}}\right)+\mathbf{1} \leqq \sum\left(\left(n_{i}+\mathbf{1}\right)-\mathbf{2}\right)+\mathbf{1}=n-\mathbf{2} .
$$

Note that the proof consists of a version of the statement that the encounter points in a rectangle can be connected to boundary points in such a way that the graph which arises is a tree, and hence that there are not enough boundary points to accommodate a positive frequency of encounter points for large rectangles. We 
have chosen the above formulation to avoid messy statements concerning paths and the corresponding visual arguments.

We list in the references a number of related articles with partially overlapping results.

Acknowledgements. We would like to express our appreciation to the SFB 170 and to the Institut für Mathematische Stochastik of the University of Göttingen for their support and hospitality. The first author was supported in addition by the Alexander von Humboldt Foundation and AFOSR Grant 88-0105.

\section{References}

1. Aizenman, M., Kesten, H., Newman, C.M.: Uniqueness of the infinite cluster and continuity of connectivity functions for short and long range percolation. Commun. Math. Phys. 111, 505-532 (1987)

2. Akcoglu, M.A., Krengel, U.: Ergodic theorems for superadditive processes. J. Reine Angew. Math. 323, 53-67 (1981)

3. Burton, R.M., Keane, M.: Topological and metric properties of infinite clustering in stationary two-dimensional site percolation (to appear)

4. Gandolfi, A.: Uniqueness of the infinite cluster for stationary finite range Gibbs states (to appear)

5. Gandolfi, A., Grimmett, G.R., Russo, L.: On the uniqueness of the infinite open cluster in the percolation model. Commun. Math. Phys. 114, 549-552 (1988)

6. Gandolfi, A., Keane, M., Russo, L.: On the uniqueness of the infinite occupied cluster in dependent two-dimensional site percolation. Ann. Prob. (to appear)

7. Grimmett, G.R.: On the number of clusters in the percolation model. J. Lond. Math. Soc. 13, 346-350 (1976)

8. Krengel, U.: Ergodic theorems, pp. 201-210. Berlin, New York: de Gruyter 1985

9. Newman, C.M., Schulman, L.S.: Infinite clusters in percolation models. J. Stat. Phys. 26, 613-628 (1981)

Communicated by M. Aizenman

Received August 30, 1988; in revised form November 14, 1988 
